\title{
A Framework for an Energy-Efficient Bandwidth Allocation Approach through Dynamic ONTs Grouping in Flexible GPON Access Networks
}

\author{
Ali A. Hammadi \\ Faculty of Electronic Engineering Technology, College of Technological Studies, The Public Authority for Applied Education and \\ Training (PAAET), Kuwait, Kuwait \\ Email: aa.hammadi@paaet.edu.kw
}

How to cite this paper: Hammadi, A.A. (2020) A Framework for an Energy-Efficient Bandwidth Allocation Approach through Dynamic ONTs Grouping in Flexible GPON Access Networks. Int. J. Communications, Network and System Sciences, 13, 1-14. https://doi.org/10.4236/ijcns.2020.131001

Received: December 16, 2019

Accepted: January 18, 2020

Published: January 21, 2020

Copyright ( 2020 by author(s) and Scientific Research Publishing Inc. This work is licensed under the Creative Commons Attribution International License (CC BY 4.0).

http://creativecommons.org/licenses/by/4.0/

(c) (i) Open Access

\begin{abstract}
The growing demands for high speed connectivity to keep pace with bandwidth intensive applications and services have spawned the idea of developing PONs with capabilities beyond those of copper and wireless-based technologies in access network. In this article, an approach for the design of an energy efficient bandwidth allocation mechanism for the shared upstream communication link in the Fiber to the Home (FTTH) access network is presented and evaluated using Mixed Integer Linear Programming (MILP) model. In the MILP model, two objective functions for minimization of power consumption and minimization of blocking were evaluated. The results have shown that with the objective of power minimization approach, Optical Network Terminals (ONTs) are efficiently grouped to the minimum number of active networking Optical Line Terminal (OLT) switches, traffic is groomed, ports are efficiently utilized, and hence total power consumption is minimized. Results have shown that with energy efficient bandwidth allocation approach consideration, energy savings can reach up to $80 \%$ for different examined traffic loads following uniform distribution.
\end{abstract}

\section{Keywords}

Fiber to the Home (FTTH), Giga Passive Optical Network (GPON), Energy Efficiency, Blocking Probability, Optimization

\section{Introduction}

The unprecedented growth in the number of internet users along with the 
emergence of a plethora new bandwidth-hungry applications and services have led to significant research efforts over the past two decades in order to set new requirements for future high-speed networks [1]. The new requirements aim to overcome many challenges and limitations that appeared in the conventional designs in access network based on copper or wireless [2]. Access network is found today as the major contributor of the total energy consumption of the global IT network infrastructure [3]. Energy concerns along with requirement of more bandwidth per user have driven the need of energy efficient high-speed access network. This came as an objective to promote FTTH deployment to reduce carbon foot print in the first place and to improve per user speed rate in the second place [2].

PONs have shown great performance compared to copper and wireless access network. PON has overcome many limitations and shortcomings appeared in conventional access network technologies [4]. PONs with its different technologies adopted such as EPON, GPON, and BPON have proven its capability in providing high per user data rate, high immunity to electromagnetic interference, high level of security, long distance reach, low cost and low energy consumption. With all these features, PONs have become a premium solution for access network design [4].

Over the last decade, many studies have been demonstrated by the FTTH European Council to predict the upstream and downstream of traffic growth. One latest study has reported that the 2020 demand estimation for downlink and uplink traffic growth is 8 Gbyte and 3 Gbyte per day respectively [5]. Asian countries have also shown radical improvement in PONs deployment. As reported by FTTH council, 61 million homes in Asia have been provided with FTTH compared to 5 million homes in Europe. Japan, Korea and China have high population densities which were a great factor in facilitating the easy deployment of FTTH [5]. Today, South Korea is leading the world in the percentage of homes with fiber connections [5].

Different studies with different methodologies and approaches appeared in previous work to improve the design of PONs network and mainly tackled two issues; efficient dynamic bandwidth allocation and power consumption. Wavelength grouping for efficient utilization of resources in PON networks has been studied thoroughly in [6]-[10]. Authors in [6]-[10] have proposed methods for the migration of low utilized ONUs to reduce the total used wavelengths and hence reduce the overall power consumption. In [11], the Software Defined Network (SDN) is integrated into the PON architecture through a central controller to dynamically provision wavelengths, timeslots, and link rates based on the traffic load. Results in [11] have shown the guaranteed quality of service and an improvement of the energy saving of the OLT power consumption for loads lower than $80 \%$. Dynamic ONUs grouping algorithm through a centralized SDN controller is introduced in [12] to further reduce overall power consumption for multiple of OLTs implementation. In the present work, mathematical optimization model using linear programming is developed to further investigate the dy- 
namic GPON infrastructure design to find the optimum result for an objective to minimize power consumption. The developed mathematical model for the minimized power consumption shall ensure that all demands are satisfied, resources are efficiently assigned, and energy consumption is minimized.

In this paper, Section II shall present a review on GPON FTTH conventional network design covering concepts, technologies, and optical cable distribution network design. Problem statement, network design and the optimization approach used to solve the problem are discussed in Section III. Section IV describes the optimization mathematical model implemented with emphasis on minimization of power consumption. Section V demonstrates obtained results with technical discussion. In Section VI, the paper is concluded.

\section{Giga Passive Optical Network (GPON) System}

State of art GPON networks depicted in Figure 1 can provide triple play services for voice, data, and video services to subscribers that can be $20 \mathrm{~km}$ away from the Central Office (CO). GPON technology is designed to consist of Optical Line Terminal (OLT) located at the CO, Optical Network Units (ONUs) at the subscribers' premises, and the Passive Optical Distribution Network (ODN) [13]. Following is a description of each component of the GPON system.

\subsection{Optical Line Terminal Equipment (OLT)}

OLT switch as depicted in Figure 2 is installed at the Central Office in the exchange. The OLT switch can terminate multiple passive optical network to ONTs where end users are connected. OLT switch is also responsible for managing and controlling channel access among competing ONTs for resources. OLT is interfaced with different systems and gateways for services and applications to deliver voice, video and data to end users [13]. The OLT port downstream connection speed from OLT to ONT is $2.5 \mathrm{~Gb} / \mathrm{s}$ while the downstream speed in $1.2 \mathrm{~Gb} / \mathrm{s}$

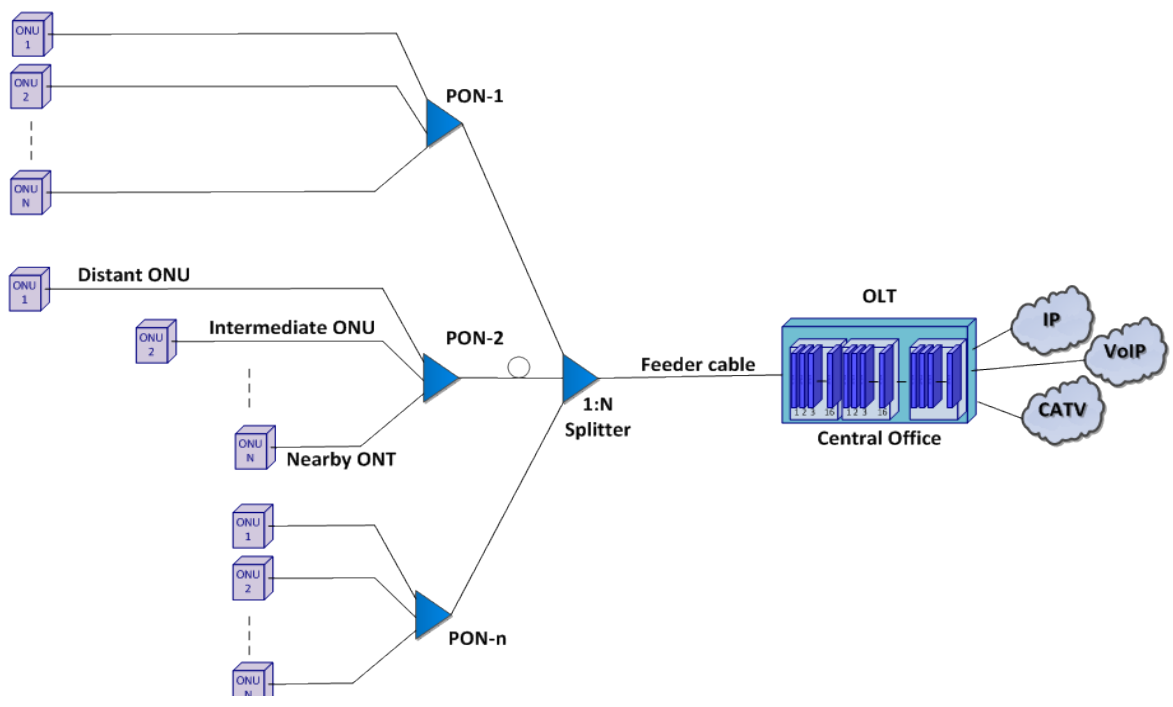

Figure 1. FTTx GPON deployment. 


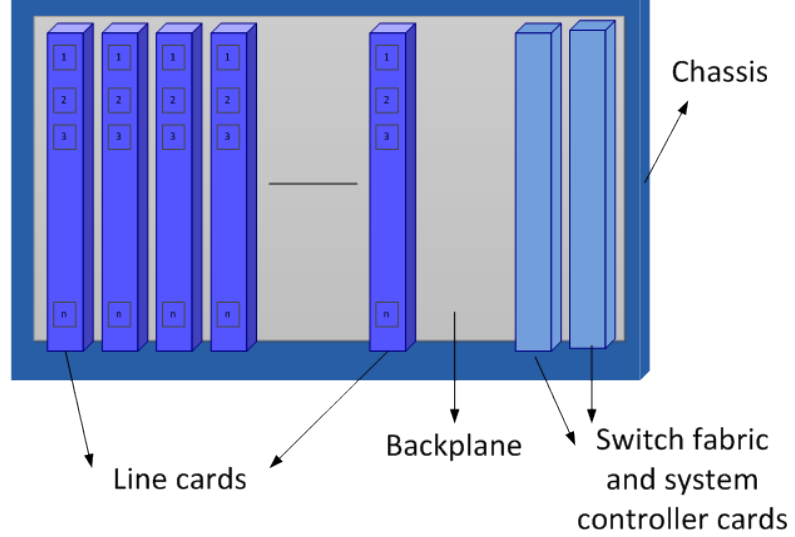

Figure 2. Optical line terminal chassis.

on each GPON Port [13]. In the Downstream side, the wavelengths assigned are 1480 are $1500 \mathrm{~nm}$ whereas in the upstream $1260-1360 \mathrm{~nm}$ is used [13].

One OLT chassis as the depicted in Figure 2 can support 16 Access Modules (AM) cards each of which is equipped with two GPON ports. Each GPON Port can support different split ratios. If the split ratio is 64, one OLT chassis with 16 cards can support 2048 subscribers.

\subsection{Optical Network Terminal (ONT)}

ONTs units are installed in each of the subscribers' premises and one ONT as shown in Figure 3 can provide 4 Telephone Lines, 2 data Ethernet ports and IP TV. There are different types of ONT for medium and large business establishment and multistoried building which varies in the number of ports. Examples of these ONTs are the SOHO which can support 8 telephone lines, and the Multi-Dwelling Unit (MDU) which consists of three units and has capability to provide 24 telephone lines and 12 high speed data connections [2].

\subsection{Fiber Optic Cables Distribution Networks}

The network cable distribution design is based on distributed splitting, where several different configurations of cascaded passive optical splitters are planned in every route to meet a pre-designed split ratio from the exchange office to end users. The cable distribution network starts from the exchange office and terminates at the end users' premises.

Four categories of fiber cables are considered in the design; Primary Optical feeder cable, Secondary Optical feeder cable, distribution Optical cable and Drop Optical cable. Figure 4 depicts a real network implementation scenario for the FTTH project [4]. Different cables are used at different segments of the network. One primary feeder cable as shown in Figure 4 with 96 strands of single mode fibers are terminated at the central office to 96 Access Module (AM) ports at the OLT switches. The cable is laid using the shortest route passing through all the sub-areas. Figure 4 demonstrates 3 locations of a total of 4 locations along the 


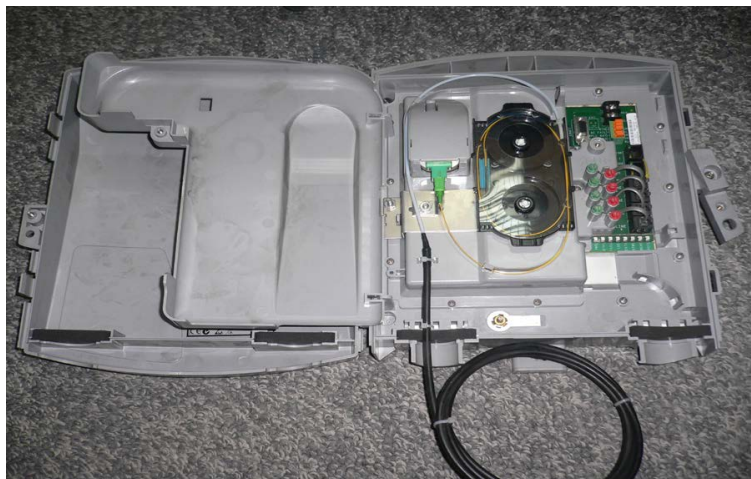

Figure 3. Optical Network Terminal (ONT).

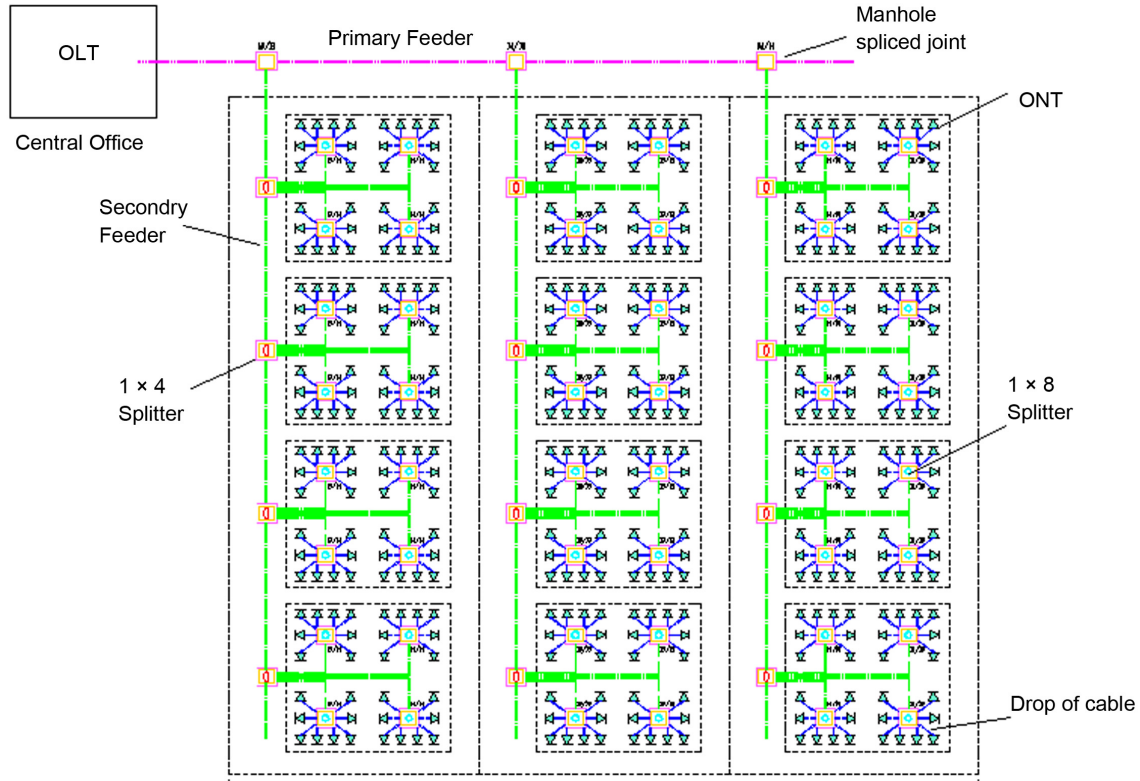

Figure 4. PON cable network distribution.

route where 24 strands of the primary feeder optical cable are spliced with a 24-core secondary feeder fiber cable. A total of four of 24-core optical cables are jointed at 4 different intersection points to pass through the various main blocks of the sub-areas.

At the Block intersection, one fiber from each of the 24 fibers of the secondary optical fiber cable is spliced with $1 \times 4$ passive optical splitter. The four outputs of the splitter are spliced with 4 cores of a distribution cable. Each of the fibers of the distribution cable is spliced with $1 \times 8$ passive splitter located near 8 subscribers. Drop of cables is laid from each of the 8 outputs of the splitter to connect to an ONT device in the subscriber home. The output of the 1:8 splitters, a drop cable of 1 fiber strand in case of single family premise or 2 strands in case of multistoried building will be laid up to the building.

\section{Problem Statement and Modeled Network Design}

In conventional FTTx PON designs as depicted in Figure 4 [2], each PON group 
is statically connected to an OLT port. In this case, resources for each ONT within its group are reserved whether the ONT has traffic to send or not. This design has shown many limitations with respect to efficiency in assigning resources and power consumption. The design depicted in Figure 5, resolved these challenges appeared in the design depicted in Figure 4 by allowing any ONT unit to be capable of joining multiple OLT switches by tuning to a wavelength that connects with designated OLT switch. Global Fabric Manager (GFM) is the central entity and the main controller where all demand requests are forwarded. GFM has a global knowledge of network as it monitors the utilization of uplink/downlink wavelengths between PONs and OLTs, and utilizations of uplink ports between OLTs and gateways. GFM assigns transmission wavelengths and time slots within the TDM frames for transmission between PONs and OLT switches. GFM aims to assign resources to satisfy the requirement of all demands while minimizing the total number of OLT switches needed and the number of activated AM ports. With this approach applied, the network is efficiently utilizing its resources and minimizing the total power consumption at the same time.

The solver program used is CPLEX [14] [15]. Running Lenovo with core i7 CPU, with $2.8 \mathrm{GHz}$ processor and 8GB RAM. CPLEX along with AMPL are used to solve the mixed integer linear programming problem. CPLEX was developed and is maintained by IBM. AMPL was developed in Bell laboratories and is a powerful algebraic modeling language for optimization problems [14] [16].

\section{MILP Mathematical Model}

In this section, two objective functions are evaluated in the MILP optimization model; minimization of power consumption and minimization of blocking. The

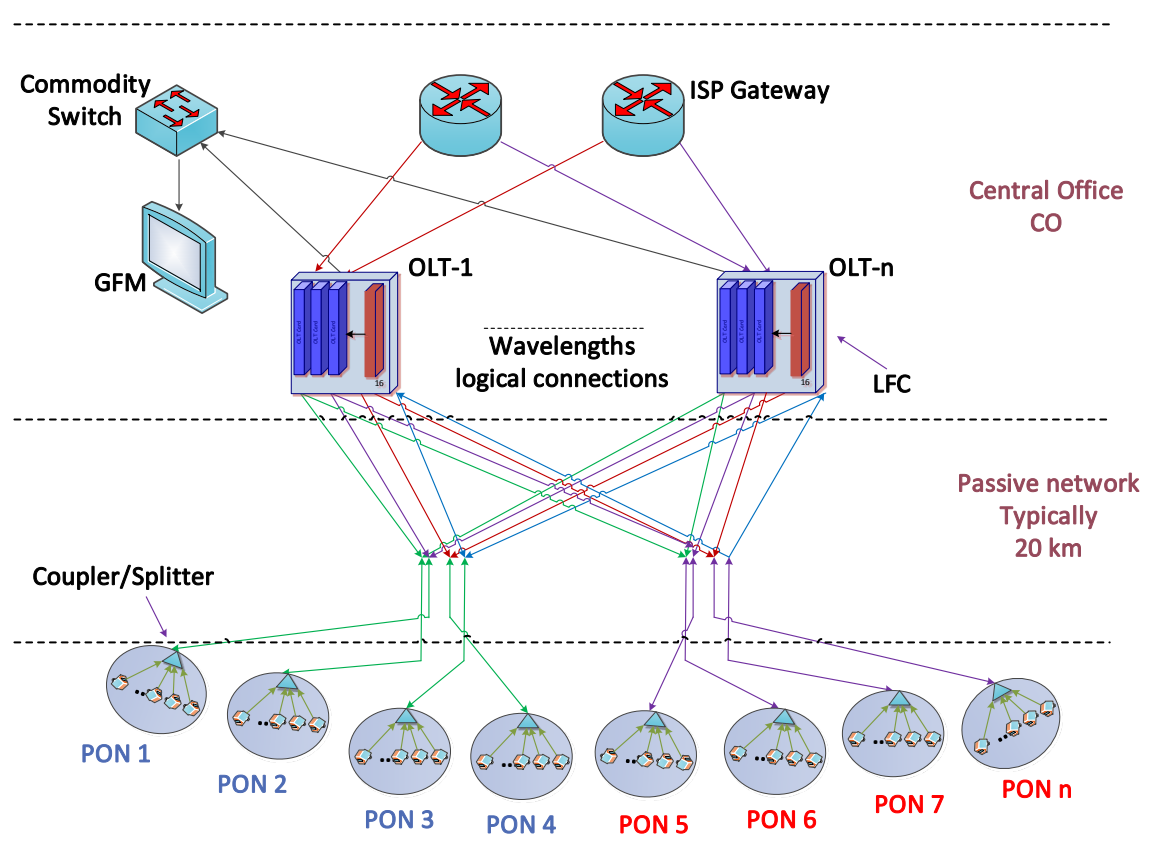

Figure 5. Conceptual design for modeled GPON network. 
objective function with the minimization of the total power consumption aims to minimize total active power consuming components needed to service all queued demands through means of dynamic bandwidth assignment and energy efficient grouping in the upstream direction from ONUs to ISPs via the minimum possible number of OLT ports and switches. Minimization of the total power consumption shall not only result in minimization of total number of access modules (AM) ports and OLT switches but also will result in maximization of throughput in the upstream direction through efficient utilization and assignment of uplink resources. Figure 6 depicts a conceptual design of the GPON modeled network.

The second objective function with the minimization of total blocked demands aims to minimize the total number of rejected requests and hence maximize the total number of served demands.

The parameters and variables used in the model are defined in Table 1 and Table 2 respectively.

Before introducing the constraints and quantities used in the model, evaluated objective functions are defined as.

\subsection{Minimize Power Consumption Objective}

$$
\sum_{i \in T} \gamma^{i}(\Phi+\nabla)+\Gamma \sum_{i \in P} \sum_{j \in A}\left(\left(\sum_{s \in P} \sum_{d \in G} \lambda_{i j}^{s d}\right) / C\right)+\varphi \sum_{i \in O} \beta^{i}
$$

The objective function with the minimization of the total power consumption aims to minimize total active power consumption from components such as total number of active OLT switches, controller cards, total number of OLT cards, total number of AM ports, and total number of active ONT units.

\subsection{Minimize Blocking Objective}

$$
\sum_{s \in P} \sum_{d \in G} \Lambda^{s d}
$$

The objective function with the minimization of total blocked demands aims to minimize the total number of rejected requests to maximize the total number

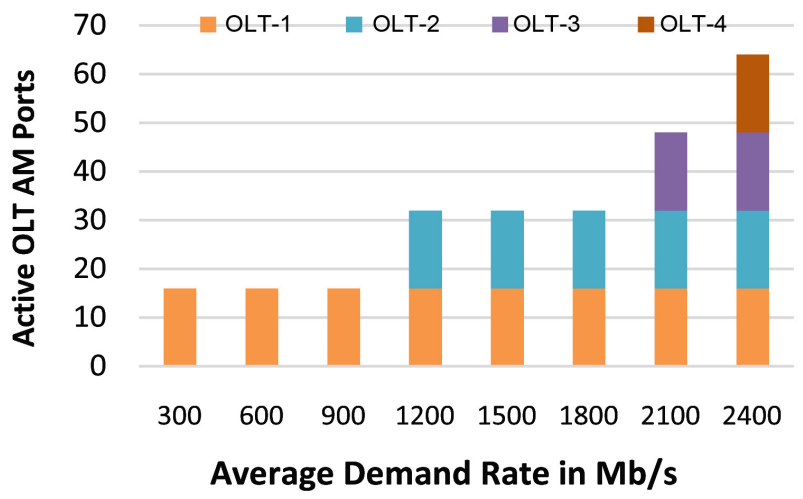

Figure 6. Total number of active OLT ports for the minimized power consumption objective. 
Table 1. Parameter.

\begin{tabular}{cl}
\hline$N$ & Set of all vector nodes. \\
$N_{X}$ & Set of neighbor nodes of node $x$, where $(x \in N)$. \\
$T$ & Set of OLTs. \\
$P$ & Set of PONs representing a splitter/coupler connecting group of homes. \\
$G$ & Set of Gateways at central office connecting OLTs with ISPs. \\
$O$ & Set of ONT Units connecting homes. \\
$A$ & Set of access ports. \\
$C$ & Wavelength capacity in the upstream flow from a PON to an OLT port. \\
$\lambda^{i j}$ & Data rate demand between every pair of source and destination $(i, j)$. \\
$\varphi$ & Denotes power consumption of an ONU. \\
$\Phi$ & $\quad$ Denotes power consumption of an OLT chassis. \\
$\nabla$ & Denotes power consumption of OLT's controller card (Local Manager). \\
$\Gamma$ & Denotes power consumption of an OLT AM port. \\
$\mathcal{M}$ & $\quad$ Large Multiplication constant, $\mathcal{M}=1000$. \\
$K$ & $\quad$ Small Multiplication constant, $K=10$ \\
$(s, d)$ & Denotes source and destination vector nodes. \\
$(x, n)$ & Denotes end points of a physical link.
\end{tabular}

Table 2. Variables.

\begin{tabular}{cl}
\hline$\lambda_{x n}^{s d}$ & Portion of traffic demand $(s, d)$ traversing physical link $(x, n)$. \\
$\gamma^{i}$ & Binary variable equals 1 to indicate OLT switch is active, otherwise equal 0. \\
$\Lambda^{s d}$ & Binary variable equals 1 if demand $L^{s d}$ is not served, otherwise equals 0. \\
$\zeta^{i j}$ & Binary variable equals 1 to indicate if OLT port is active, otherwise equals 0. \\
$\beta^{i}$ & Binary variable equals 1 to indicate if ONT is active, otherwise equals 0. \\
$\psi^{i}$ & Number of activated ports in OLT switch $i$. \\
\hline
\end{tabular}

of served demands.

These objective functions are subject to the below constraints.

\subsection{Flow Conservation Constraint}

$$
\sum_{\substack{n \in N_{x} \\
x \neq n}} \lambda_{x n}^{s d}-\sum_{\substack{n \in N_{x} \\
x \neq n}} \lambda_{n x}^{s d}=\left\{\begin{array}{ll}
\lambda^{s d}\left(1-\Lambda^{s d}\right) & x=s \\
-\lambda^{s d}\left(1-\Lambda^{s d}\right) & x=d \quad \\
0 & \text { otherwise }
\end{array} \quad \forall s \in P, \forall d \in G \text { and } \forall x \in N\right.
$$

Constraint (3) is the flow conservation constraint. It ensures that the total traffic going into a node is equal to the total traffic leaving it for all nodes except the source and destination of a demand.

\subsection{Upstream Link Capacity Constraint}

$$
\sum_{s \in P} \sum_{d \in G} \lambda_{i j}^{s d} \leq C \quad \forall i \in P \text { and } \forall j \in A
$$


Constraint (4) ensures that the upstream traffic shall not exceed the capacity of the uplink wavelength between PONs and OLT ports.

\subsection{Upstream Wavelength's Utilization Constraint}

$$
\left(\sum_{s \in P} \sum_{d \in G} \lambda_{i j}^{s d}\right) / C \leq 1 \quad \forall i \in P \text { and } \forall j \in A
$$

Constraint (5) ensures that the utilization of the uplink wavelengths between PONs and OLT ports are not exceeded.

\subsection{Demand Satisfaction Constraint}

$$
\sum_{s \in P} \sum_{d \in G} \lambda^{s d} \leq \sum_{x \in T} \sum_{s \in P} \sum_{d \in G} \sum_{n \in N_{x}} \lambda_{n x}^{s d}
$$

Constraint (6) ensures that the total number of queued demands at the ONTs is equal to the total number of served demands by the OLTs. This constraint is enforced to prevent blocking of any demand to occur.

\subsection{Total Number of Active Switched OLTs}

$$
\begin{gathered}
\sum_{s \in P} \sum_{d \in G} \sum_{n \in N_{x}} \lambda_{n x}^{s d} \geq \gamma^{x} \\
\mathcal{M} \gamma^{x} \geq \sum_{s \in P} \sum_{d \in G} \sum_{n \in N_{x}} \lambda_{n x}^{s d} \\
\text { Tot_Num_OLTs }=\sum_{x \in T} \gamma^{x} \\
\forall x \in T
\end{gathered}
$$

Constraints (7) and (8) ensures that assigned OLT switches to serve ONTs are switched on, while Equation (9) counts the total number of active OLT switches.

\subsection{Total Number of AM OLT Ports Constraint}

$$
\begin{gathered}
\left(K\left(\sum_{s \in P} \sum_{d \in G} \lambda_{x j}^{s d}\right) / C\right) \geq \zeta^{x j} \\
\left(K\left(\sum_{s \in P} \sum_{d \in G} \lambda_{x j}^{s d}\right) / C\right) \leq \mathcal{M} \zeta^{x j} \quad \forall x \in P \& \forall j \in T \\
\psi^{x}=\sum_{x \in P} \zeta^{x j} \quad \forall j \in T \\
\psi^{x} \leq \chi \quad \forall x \in T
\end{gathered}
$$

Constraints (10) and (11) ensures used AM ports in OLT Switches are switched on, and constraint (13) ensures total number of active AM ports calculated in Equation (12) does not exceed total number of AM ports $(\chi)$ in OLT switch.

\section{Results and Discussions}

In this work, two objective functions are compared. First objective aims to mi- 
nimize total power consumption through efficient grouping of ONT units to minimum possible number of OLT switches and AM ports. This allows the switch off of not used or idle switches and ports within the network. The second objective function is the objective with minimization of blocking with a constraint to satisfy all demand. There is no emphasis of the second objective model on the mechanism of how and when the resources are assigned. Hence, the resources are assigned in random manner.

The OLT switch used in the model is the Cisco ME4600 [17] [18]. The chassis power consumption of the OLT switch is $60 \mathrm{~W}$, while the Local Fabric Controller (LFC) consumes $180 \mathrm{~W}$. The power consumption of AM module of the Cisco ME4600 at full load is $90 \mathrm{~W}$. The total number of GPON AM ports $(\chi)$ that can be hosted in one chassis is 256 ports. The ONT units used in the model are tunable ONTs with power consumption of $2.5 \mathrm{~W}$ at full load [19]. The evaluated demand average rate follows a uniform distribution and ranges between 300 and $2400 \mathrm{Mb} / \mathrm{s}$. Four OLT switches are considered in the modeled network and 16 PON groups. The optical distribution network is depicted in Figure 4 where at the CO, a wavelength selective switch [20] can be used for wavelength conversion to provide flexibility for the connectivity from any of the PON groups to any of the 4 OLTs.

The results in Figures 6-10 have shown that as the rate or the size of average

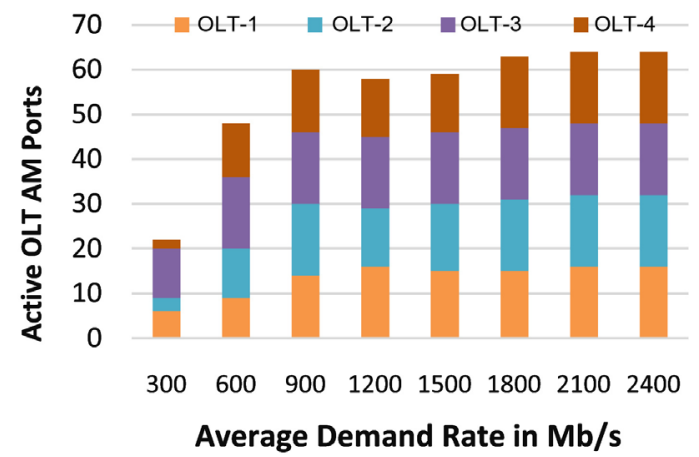

Figure 7. Total number of active OLT ports for the minimized blocking objective.

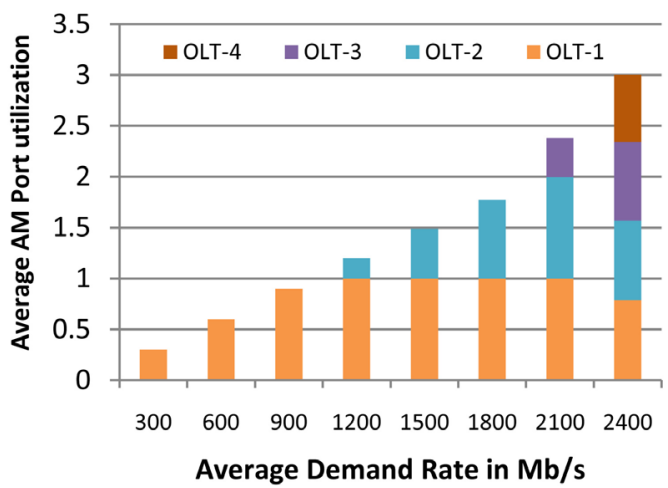

Figure 8. Average port utilization for the minimized power consumption objective. 


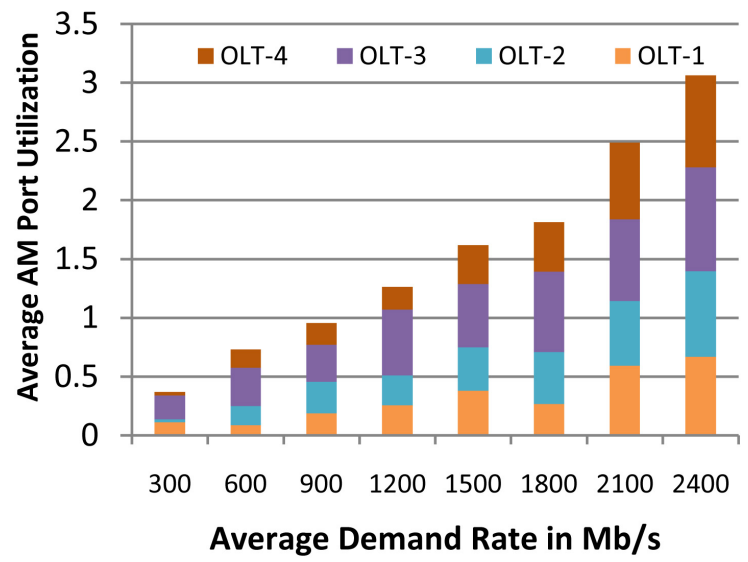

Figure 9. Average port utilization for minimization of blocking objective.

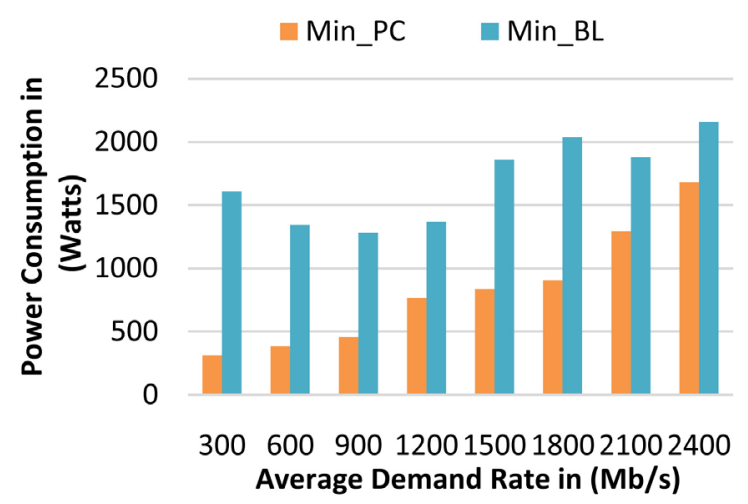

Figure 10. Total power consumption for minimization of power and minimization of blocking objectives.

demands increases, the number of activated AM ports and OLT switches increases. Hence the power consumed by the network is increasing as the size of demand is increasing. This result is common for the two objective functions. However, the percentage of increase is higher with the minimization of blocking objective as the assignment of resources with that approach is random. Random assignment is inefficient as links and hardware resources are underutilized. This can be clearly observed from Figure 7 and Figure 9. Figure 6 and Figure 7 demonstrate the total number of active OLT switches and AM ports for the minimization of power consumption and minimization of blocking objectives, respectively. Figure 6 shows that total number of active components is kept to minimum through grouping and consolidating demands to minimum possible number of OLT switches and AM ports. This approach has efficiently utilized the resources of the communication links and hardware as shown in the results of Figure 9.

On the other hand, minimization of blocking objective approach is inefficient as demands are randomly assigned to OLTs. This method provisions all ONTs with resource to communicate with ISP through OLT switches, however links and hardware resources are underutilized especially at low rates. Figure 7 and 
Figure 9 demonstrate that all OLT switches are activated for all average demand rate with low utilization.

For a comparison on the efficiency of the utilization of resources between the two objectives, number of active OLT switches evaluated with average rates between 300 and $900 \mathrm{Mb} / \mathrm{s}$ for the minimization of power consumption is 1 with average utilization ranges between $25 \%$ and $90 \%$. While the objective of minimization of blocking has shown that the number of activated OLT switches for the same average range of rates is 4 with $10 \%$ to $50 \%$ of utilization of resources.

Figure 10 demonstrates the total power consumption result for the two objective functions. Equation (1) earlier in Section 4 described the main quantities and variables that constitute the total power consumption. These values are the number of activated OLT switches, number of AM ports, number of ONTs with queued demands. Therefore; the reason behind the reduction of power consumption with the minimization of power consumption objective against the objective of minimization of blocking, is the reduced number of active OLTs and AM ports used to service all demands.

\section{Conclusion}

The rapid growth in bandwidth-hungry applications and services has set new requirements for high speed infrastructure in access network to overcome many limitations appeared in current implemented technologies. PONs in access network in the last decade were found as a premium solution to resolve many challenges appeared with the conventional wireless and copper-based designs. PONs have shown a proven performance in providing high per user access rate and reducing the overall network power consumption. In this work, a mathematical optimization approach was presented to reduce the power consumption through means of consolidation and efficient utilization of network and hardware resources. Different loads of traffic rate have been evaluated following uniform distribution. Hardware and network resources have shown efficient utilization and power savings results have reached $80 \%$ for the approach with minimization of power consumption objective when compared with conventional approaches in network designs such as the approach of minimization of blocking. The work presented in this article has been limited to mathematical models. However, for a future work, computer simulations to design heuristics to validate the results obtained from the MILP mathematical models shall be of great interest.

\section{Conflicts of Interest}

The author declares no conflicts of interest regarding the publication of this paper.

\section{References}

[1] Hammadi, A. and Mhamdi, L. (2014) Review: A Survey on Architectures and Energy Efficiency in Data Center Networks. Computer Communication, 40, 1-21. https://doi.org/10.1016/j.comcom.2013.11.005 
[2] Hammadi, A., Talwar, R. and Singh, H. (2017) A Case Study on the Architecture, Design, Implementation and Testing of Fiber to the Home (FTTH) Green Network in Kuwait. International Journal of Current Engineering and Technology, 7, 1622-1631.

[3] Baliga, J., Ayre, R., Sorin, W.V., Hinton, K. and Tucker, R.S. (2008) Energy Consumption in Access Networks. Optical Fiber Communication/National Fiber Optic Engineers Conference, San Diego, 24-28 February 2008, 1-3. https://doi.org/10.1109/OFC.2008.4528538

[4] Hammadi, A.A. (2016) Future PON Data Centre Networks. University of Leeds, School of Electronic and Electrical Engineering, Leeds.

[5] The Growth of Fiber to the Home. http://www.ftthcouncil.eu

[6] Bakarman, H.A., Shaari, S. and Ismail, M. (2010) Simulation of 1.25 Gb/s Downstream Transmission Performance of GPON-FTTx. International Conference on Photonics, Langkawi, 5-7 July 2010, 1-5. https://doi.org/10.1109/ICP.2010.5604440

[7] Wang, W., Guo, W., Li, C., Hu, W. and Xia, M. (2017) ONU Aggregation Schemes for TWDM PONS with Multiple Tuning Ranges. IEEE/OSA Journal of Optical Communications and Networking, 9, 319-326.

https://doi.org/10.1364/JOCN.9.000319

[8] Luo, Y., Sui, M. and Effenberger, F. (2012) Wavelength Management in Time and Wavelength Division Multiplexed Passive Optical Networks (TWDM-PONs). IEEE Global Communications Conference (GLOBECOM), Anaheim, 3-7 December 2012, 2971-2976. https://doi.org/10.1109/GLOCOM.2012.6503569

[9] Yang, H., Sun, W., Li, J. and Hu, W. (2014) Energy Efficient TWDM Multi-PON System with Wavelength Relocation. Journal of Optical Communications and Networking, 6, 571-577. https://doi.org/10.1364/JOCN.6.000571

[10] Wang, R., Lee, H.H., Lee, S.S. and Mukherjee, B. (2014) Energy Saving via Dynamic Wavelength Sharing in TWDM-PON. IEEE Journal on Selected Areas in Communications, 32, 1566-1574. https://doi.org/10.1109/JSAC.2014.2335332

[11] Pakpahan, A.F., Hwang, I. and Nikoukar, A. (2017) OLT Energy Savings via Software-Defined Dynamic Resource Provisioning in TWDM-PONs. IEEE/OSA Journal of Optical Communications and Networking, 9, 1019-1029. https://doi.org/10.1364/JOCN.9.001019

[12] Gu, R., Zhang, S., Li, H. and Ji, Y. (2014) Dynamic ONU Grouping in Flexible Passive Optical Networks. 2014 International Conference on Optical Network Design and Modeling, Stockholm, 19-22 May 2014, 210-215.

[13] Keiser, G. (2006) FTTX-Concepts and Applications. John Wiley \& Sons Ltd., Hoboken. https://doi.org/10.1002/047176910X

[14] I. I. CPLEX (2009) V12.1: User's Manual for CPLEX. Vol. 46, International Business Machines Corporation, Armonk, 157.

[15] ILOG CPLEX: High-Performance Software for Mathematical Programming and Optimization. http://www.ilog.com/products/cplex

[16] Pioro, M. and Medhi, D. (2004) Routing, Flow and Capacity Design in Communication and Computer Networks. Morgan Kaufmann, San Francisco. https://doi.org/10.1016/B978-012557189-0/50011-1

[17] Hammadi, A. (2017) Mathematical Optimization Modelling for Fast-Switched and Delay Minimized Scheduling for Intra-Cell Communication in an AWGR-Based PON Data Center. International Journal of Communications, Network and System Sciences, 10, 13-29. https://doi.org/10.4236/ijcns.2017.102002

[18] Cisco: Data Sheet of Cisco-ME 4600 Series Optical Line Terminal Data Sheet. 
[19] Grobe, K., Roppelt, M., Autenrieth, A., Elbers, J.P. and Eiselt, M. (2011) Cost and Energy Consumption Analysis of Advanced WDM-PONs. Communications Magazine, 49, s25-s32. https://doi.org/10.1109/MCOM.2011.5706310

[20] Anderson, R. (2003) US Patent 6.542,657: Binary Switch for an Optical Wavelength Router. 